\title{
Relations between Entanglement Witnesses and Bell Inequalities
}

\author{
Philipp Hyllus, ${ }^{1}$ Otfried Gühne, ${ }^{2}$ Dagmar Bruß,${ }^{1,3}$ and Maciej Lewenstein $* 1,4$ \\ ${ }^{1}$ Institut für Theoretische Physik, Universität Hannover, D-30167 Hannover, Germany. \\ ${ }^{2}$ Institut für Quantenoptik und Quanteninformation, \\ Österreichische Akademie der Wissenschaften, A-6020 Innsbruck, Austria. \\ ${ }^{3}$ Institut für Theoretische Physik III, Universität Düsseldorf, D-40225 Düsseldorf, Germany. \\ ${ }^{4}$ ICFO - Institut de Ciències Fotòniques, 08034 Barcelona, Spain.
}

(Dated: August 28, 2018)

\begin{abstract}
Bell inequalities, considered within quantum mechanics, can be regarded as non-optimal witness operators. We discuss the relationship between such Bell witnesses and general entanglement witnesses in detail for the Bell inequality derived by Clauser, Horne, Shimony, and Holt (CHSH). We derive bounds on how much an optimal witness has to be shifted by adding the identity operator to make it positive on all states admitting a local hidden variable model. In the opposite direction, we obtain tight bounds for the maximal proportion of the identity operator that can be subtracted from such a CHSH witness, while preserving the witness properties. Finally, we investigate the structure of CHSH witnesses directly by relating their diagonalized form to optimal witnesses of two different classes.
\end{abstract}

PACS numbers: 03.65.Ud, 03.67.-a

\section{INTRODUCTION}

One of the most remarkable features that distinguishes quantum mechanics from classical mechanics is entanglement, i.e., quantum correlations between separated physical systems that can be stronger than correlations allowed by classical mechanics. Bell inequalities [1] bound the correlations within any local and realistic theory. In a local theory, measurement outcomes cannot depend on the choice of measurement direction of another spacelike separated observer, while in a realistic theory, the results of any measurement are predetermined, regardless of whether the measurement is carried out or not. These Bell inequalities are violated by certain entangled states so that quantum mechanics cannot be regarded as a local and realistic theory.

The original Bell inequality [1], which is based on the perfect anti-correlations of the so-called singlet state, was later extended by Clauser, Horne, Shimony, and Holt (CHSH) 2] to a more general inequality for two observers each having the choice of two measurement settings with two outcomes. In the following years, several generalizations of the CHSH inequality have been derived. Inequalities for $n$ observers, each having at their disposal two dichotomic measurements (i.e. measurements with two outcomes) per site were studied by Mermin [3], Ardehali [4], Belinskii and Klyshko [5]. The complete set of such inequalities was finally constructed by Werner and Wolf [6] and independently by Żukowski and Brukner [7]. Further, generalizations to more outcomes [8] and to several settings per site have been made, see, for instance, Refs. 9, 10, 11, 12. . Recently, the non-locality of quantum states was studied also from a different perspective

\footnotetext{
*also at Instituciò Catalana de recerca i estudis avançats.
}

besides Bell inequalities. Namely the question whether a quantum state can be simulated by so-called non-local machines was investigated [13, 14, 15].

The violation of a Bell inequality implies the nonexistence of a local hidden variable (LHV) model for the correlations observed with respect to a certain state [16]. In the following, when we say that a state admits a LHV model, it is understood that this model is constructed with respect to a particular Bell inequality, with a fixed number of measurement settings per observer and with a fixed number of outcomes of each setting.

In this paper we systematically investigate the relation between the CHSH inequality and entanglement witnesses, or, more precisely, optimal entanglement witnesses. Before we introduce witnesses, we remind the reader of the precise definition of entanglement. A quantum state $\rho$ of a system composed of two subsystems of dimension $N$ and $M$, respectively, is called entangled iff it cannot be written as a separable state of the form 17]

$$
\sigma_{s}=\sum_{k} p_{k}\left|\psi_{k}\right\rangle\left\langle\psi_{k}|\otimes| \phi_{k}\right\rangle\left\langle\phi_{k}\right|,
$$

where $p_{k} \geq 0$ and $\sum_{k} p_{k}=1$. The most prominent criterion for deciding whether a given state is entangled or not is related to the partial transpose, which is defined in a real orthonormal basis as follows

$$
\begin{aligned}
\rho_{A}^{T} & =\sum_{i j k l}\langle i j|\rho| k l\rangle(|i\rangle\langle k|)^{T} \otimes|j\rangle\langle l| \\
& =\sum_{i j k l}\langle i j|\rho| k l\rangle|k\rangle\langle i|\otimes| j\rangle\langle l| .
\end{aligned}
$$

Separable states have a positive (semi-definite) partial transpose (PPT), hence all non-PPT states are entangled [18]. However, for systems of more than three parties, or for dimensions higher than $2 \times 2$ and $2 \times 3$, there exist PPT-entangled states [19]. 
Entanglement witnesses are operators that are designed directly for distinguishing between separable and entangled states [19, 20, 21]: a Hermitean operator $W$ is called an entanglement witness if it has a positive expectation value with respect to all separable states, $\operatorname{Tr}\left[W \sigma_{s}\right] \geq 0$, while there exists at least one state $\rho$ such that $\operatorname{Tr}[W \rho]<0$. The negative expectation value is hence a signature of entanglement, and a state with $\operatorname{Tr}[W \rho]<0$ is said to be detected by the witness. The concept of entanglement witnesses has turned out to be extremely important since it can be shown that for every entangled state there exists a witness detecting it. Moreover, witnesses provide a very useful tool for the experimental detection of entanglement [22, 23].

The separable states do not violate any Bell inequality. Surprisingly, the natural assumption that all entangled states do violate a Bell inequality is not true: For a one parameter family of $U \otimes U$ invariant states in $d \times d$ dimensions, where $U$ is a unitary operator, Werner constructed a LHV model for a parameter range, where the states are entangled [17, 24]. Further, it has been shown that all the inequalities for $n$ sites with two dichotomic measurement settings per site, are not violated by PPT entangled states [6], and no example for a violation of a Bell inequality by a PPT entangled state is known.

Because of the fact that they are not capable of detecting all entangled states, Bell inequalities can be regarded as non-optimal witness operators. Hence the question about the relation of witness operators and Bell inequalities concerns the relation of the border between separable and entangled states, and the border between LHV and non-LHV states. A schematic view of the different possibilities for the correlations of fixed measurement settings is given in Fig. 1.

This relation between Bell inequalities and witnesses was first studied in Ref. [20], where a so-called weak Bell inequality was introduced. Usually, Bell inequalities are required to be fulfilled at least on all uncorrelated probability distributions for the outcomes of the measurements. Weak Bell inequalities are only required to hold at least on uncorrelated probability distributions which are compatible with quantum mechanics. It turns out that these correspond to quantum mechanical product states; convex combinations of projectors onto these are separable states. Thus, weak Bell inequalities can detect all entangled states [25].

The main difficulty in the study of the relation between Bell inequalities and witnesses is the very large number of degrees of freedom of the Bell inequalities, because only the number of measurement settings per site, and the number of measurement outcomes on each site is fixed, but not the measurement settings themselves. In contrast, if all the measurement settings are fixed, then it is possible to directly apply the formalism of Ref. [21] to derive the correspondence between optimal entanglement witnesses and the Bell inequality. This was used in Ref. 26] to show that for certain fixed settings Bell inequalities for systems of two qutrits [8] correspond to

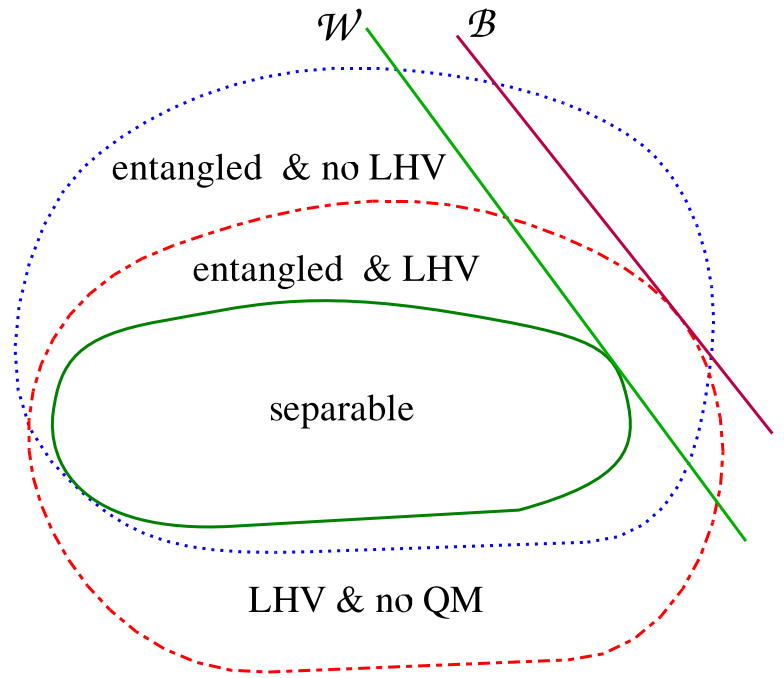

FIG. 1: Schematic picture of the different types of correlations. Generally, given a probability distribution for the outcomes of fixed measurement settings, two questions can be posed. First, one may ask whether the correlations of the distribution can origin from quantum mechanics. Second, one may ask whether they may origin from a LHV model. The figure shows the resulting four sets in the space of probability distributions: There are correlations resulting from entangled states which are not compatible with LHV models, entangled states admitting a LHV model, separable states, and correlations which may originate from LHV models, but not from quantum mechanics. $\mathcal{W}$ denotes a possible witness (the line corresponds to the hyperplane for which the expectation value $\langle\mathcal{W}\rangle$ vanishes) and $\mathcal{B}$ a Bell inequality.

decomposable witnesses and hence are not violated by PPT entangled states. For fixed measurement settings, it is also possible to relate Bell inequalities for the class of, so-called, graph states to entanglement witnesses [27].

In this paper, we will neither restrict the LHV models, nor fix the settings when treating the CHSH inequality. Our paper is organized as follows: In Section II, we recall some facts concerning witnesses and the CHSH inequality, and show how to write a CHSH inequality as a CHSH witness. Then, in Section III, we transform optimal witnesses - by adding an appropriate constant - to witnesses that detect only states which violate a $\mathrm{CHSH}$ inequality. In Section IV, we transform CHSH witnesses in the same spirit by subtracting the identity operator, bringing them closer to the set of separable states. Then we use another approach to relate the CHSH witnesses to optimal witnesses directly, by considering the diagonalized CHSH witness in Section V. Finally, we conclude and pose open questions in Section VI. 


\section{BASIC DEFINITIONS}

\section{A. The CHSH inequality}

Assume that a source emits two particles in different directions, one particle to each of two receivers, and that the receivers can perform one out of two dichotomic measurements $\hat{A}_{1,2}$ and $\hat{B}_{1,2}$, respectively. Then, if the physical process can be described by a LHV model, the inequality

$$
\left|E\left(A_{1}, B_{1}\right)+E\left(A_{1}, B_{2}\right)+E\left(A_{2}, B_{1}\right)-E\left(A_{2}, B_{2}\right)\right| \leq 2,
$$

has to be fulfilled, where $E\left(A_{i}, B_{j}\right)$ is the expectation value of the correlation experiment $\hat{A}_{i} \hat{B}_{j}$. This is the CHSH inequality [2] that gives a bound on any LHV theory trying to explain the results.

Within quantum mechanics, one can introduce the $\mathrm{CHSH}$ operator

$$
\mathcal{B}=\mathbf{a} \cdot \boldsymbol{\sigma} \otimes\left(\mathbf{b}+\mathbf{b}^{\prime}\right) \cdot \boldsymbol{\sigma}+\mathbf{a}^{\prime} \cdot \boldsymbol{\sigma} \otimes\left(\mathbf{b}-\mathbf{b}^{\prime}\right) \cdot \boldsymbol{\sigma} .
$$

Here, $\mathbf{a}=\left(a_{x}, a_{y}, a_{z}\right)$, etc., are unit vectors describing the measurements that the parties A and B perform, $\boldsymbol{\sigma}=$ $\left(\sigma_{x}, \sigma_{y}, \sigma_{z}\right)$ is the vector of Pauli operators, and $\mathbf{a} \cdot \boldsymbol{\sigma}=$ $\sum_{i} a_{i} \sigma_{i}$. The $\mathrm{CHSH}$ inequality requires that

$$
\left|\operatorname{Tr}\left[\mathcal{B} \rho_{\text {LHV }}\right]\right| \leq 2
$$

is fulfilled for all states $\rho_{\text {LHV }}$ admitting a LHV model.

A necessary and sufficient criterion for the violation of a CHSH inequality was found by the Horodeckis 28]. For stating this we need that any two qubit state can be written as

$$
\rho=\frac{1}{4} \sum_{i=0}^{3} \lambda_{i j} \sigma_{i} \otimes \sigma_{j}
$$

where $\sigma_{0}=\mathbb{1}$ and the other $\sigma_{i}$ correspond to the Pauli matrices. In the following, we will refer to the $3 \times 3$ dimensional subtensor $\lambda_{i>0, j>0} \equiv T_{\rho}$ as the correlation tensor. This tensor holds all the information that is needed to decide whether a state violates a $\mathrm{CHSH}$ inequality: A state $\rho$ violates a CHSH inequality iff $u_{1}+u_{2}>1$, where $u_{1}$ and $u_{2}$ are the two largest eigenvalues of $U_{\rho}=T_{\rho}^{T} T_{\rho}$ [28].

\section{B. Witnesses}

Let us now note some facts concerning entanglement witnesses. In systems of two qubits all entanglement witnesses are decomposable, i.e., of the form

$$
W=P+Q^{T_{A}}
$$

where $P$ and $Q$ are positive semi-definite operators [21]. Decomposable witnesses cannot detect PPT entangled states $\varrho$, which can be shown by using the identity
$\operatorname{Tr}\left[A^{T_{A}} B\right]=\operatorname{Tr}\left[A B^{T_{A}}\right]$, and the fact that the product of two positive operators remains positive: $\operatorname{Tr}[W \varrho]=$ $\operatorname{Tr}[P \varrho]+\operatorname{Tr}\left[Q \varrho^{T_{A}}\right] \geq 0$ because $\varrho^{T_{A}} \geq 0$ by assumption. It can be shown that in systems of two qubits there are only decomposable witnesses.

The optimal entanglement witnesses for two qubits are of the form $W=|\phi\rangle\left\langle\left.\phi\right|^{T_{A}}\right.$, where $\left.\mid \phi\right\rangle$ is an entangled state vector. An optimal witness detecting the entangled state $\rho$ can be constructed from the eigenvector $|\phi\rangle$ of $\rho^{T_{A}}$ with negative eigenvalue $\lambda$ as $W=|\phi\rangle\left\langle\left.\phi\right|^{T_{A}}\right.$ because $\operatorname{Tr}\left[|\phi\rangle\left\langle\left.\phi\right|^{T_{A}} \rho\right]=\operatorname{Tr}\left[|\phi\rangle\langle\phi| \rho^{T_{A}}\right]=\lambda<0\right.$. Using the same argument as above one can see that it is positive on separable states. Further, writing $|\phi\rangle$ in the Schmidt form

$$
|\phi\rangle=\alpha|00\rangle+\beta|11\rangle, \quad \alpha, \beta>0, \quad \alpha^{2}+\beta^{2}=1,
$$

the witness can be locally decomposed as [29]

$$
\begin{aligned}
W_{\alpha}= & \frac{1}{4}\left(\mathbb{1} \mathbb{1}+\sigma_{z} \sigma_{z}+\left(\alpha^{2}-\beta^{2}\right)\left(\sigma_{z} \mathbb{1}+\mathbb{1} \sigma_{z}\right)\right. \\
& \left.+2 \alpha \beta\left(\sigma_{x} \sigma_{x}+\sigma_{y} \sigma_{y}\right)\right) .
\end{aligned}
$$

Here and in the following we leave out the tensor product symbols.

We define as a CHSH witness the witness which is positive on all LHV states and which can be constructed as 20]

$$
W_{\mathrm{CHSH}}=2 \cdot \mathbb{1}+\mathcal{B} .
$$

From the definition of optimal witnesses and the $\mathrm{CHSH}$ operator it follows directly that $\mathrm{CHSH}$ witnesses cannot be optimal witnesses: The partially transposed $\mathrm{CHSH}$ witness $W_{\mathrm{CHSH}}^{T_{A}}$ is still a CHSH witness, because it transforms the CHSH operator from Eq. (4) into another CHSH operator with $a_{y} \rightarrow-a_{y}$ and $a_{y}^{\prime} \rightarrow-a_{y}^{\prime}$. However, for every optimal witness, $W_{\mathrm{opt}}^{T_{A}}$ is a positive operator. Hence $W_{\mathrm{CHSH}}$ cannot be optimal. In the following, we will investigate the relation between optimal witnesses and $\mathrm{CHSH}$ witnesses in detail.

\section{FROM OPTIMAL WITNESSES TO CHSH INEQUALITIES}

In this Section, we pose the following question: Given an optimal entanglement witness $W=|\phi\rangle\left\langle\left.\phi\right|^{T_{A}}\right.$, how much do we have to shift it by adding the identity operator such that it is positive on all states admitting a local hidden variable model? In other words, for which $\gamma>0$ is $W+\gamma \mathbb{1}$ a CHSH witness? We calculate bounds on $\gamma$, first considering witnesses where $|\phi\rangle$ is a maximally entangled state and then optimal witnesses constructed with arbitrary entangled states.

If $|\phi\rangle=\left|\phi^{+}\right\rangle$, where $\left|\phi^{+}\right\rangle$is one of the Bell states

$$
\begin{aligned}
\left|\phi^{ \pm}\right\rangle & =\frac{1}{\sqrt{2}}(|00\rangle \pm|11\rangle), \\
\left|\psi^{ \pm}\right\rangle & =\frac{1}{\sqrt{2}}(|01\rangle \pm|10\rangle),
\end{aligned}
$$


the optimal witness takes the simple local form

$$
W=\frac{1}{4}\left(\mathbb{1} \mathbb{1}+\sigma_{x} \sigma_{x}+\sigma_{y} \sigma_{y}+\sigma_{z} \sigma_{z}\right) .
$$

Now we can use the observation that

$$
\begin{aligned}
\sigma_{x} \sigma_{x}+\sigma_{y} \sigma_{y}= & \frac{1}{\sqrt{2}}\left[\sigma_{x}\left(\frac{\sigma_{x}+\sigma_{y}}{\sqrt{2}}\right)+\sigma_{x}\left(\frac{\sigma_{x}-\sigma_{y}}{\sqrt{2}}\right)\right. \\
& \left.+\sigma_{y}\left(\frac{\sigma_{x}+\sigma_{y}}{\sqrt{2}}\right)-\sigma_{y}\left(\frac{\sigma_{x}-\sigma_{y}}{\sqrt{2}}\right)\right] \\
\equiv & \frac{1}{\sqrt{2}} \mathcal{B}_{x, y}
\end{aligned}
$$

to write the witness in terms of CHSH operators as follows

$$
W=\frac{1}{4}\left(\mathbb{1} \mathbb{1}+\frac{1}{2 \sqrt{2}}\left(\mathcal{B}_{x, y}+\mathcal{B}_{x, z}+\mathcal{B}_{y, z}\right)\right) .
$$

The expectation value of each of these CHSH operators is bounded by -2 from below for states admitting a LHV model, so that we can estimate

$$
\operatorname{Tr}\left[W \rho_{\mathrm{LHV}}\right] \geq \frac{1}{4}\left(1+\frac{1}{2 \sqrt{2}}(-3 \cdot 2)\right) \equiv-\gamma .
$$

Hence, $W^{\prime}=W+\gamma \cdot \mathbb{1}$ corresponds to a CHSH witness, being positive not only on separable, but more general on all states fulfilling the CHSH inequality. The spectral decomposition of $W^{\prime}$ is given by

$$
\begin{aligned}
W^{\prime}= & \left(\frac{1}{2}+\gamma\right)\left[|00\rangle\left\langle 00|+| \psi^{+}\right\rangle\left\langle\psi^{+}|+| 11\right\rangle\langle 11|\right] \\
& -\left(\frac{1}{2}-\gamma\right)\left|\psi^{-}\right\rangle\left\langle\psi^{-}\right|,
\end{aligned}
$$

and since $1 / 2-\gamma \approx 0.220>0, W^{\prime}$ is still detecting states.

Let us estimate the strength of the witness by looking at the following family of states

$$
\rho_{p}=p|\psi\rangle\langle\psi|+\frac{(1-p)}{4} \mathbb{1},
$$

where $|\psi\rangle=a|01\rangle-b|10\rangle$, and $a, b \geq 0$. In the following, we abbreviate $x=a b$. For this family of states, the only eigenvector with possibly negative eigenvalue is $\left|\phi^{+}\right\rangle$,

$$
\rho_{p}^{T_{A}}\left|\phi^{+}\right\rangle=\left(-p x+\frac{(1-p)}{4}\right)\left|\phi^{+}\right\rangle .
$$

Hence the original witness $\left|\phi^{+}\right\rangle\left\langle\left.\phi^{+}\right|^{T_{A}}\right.$ is a good witness for these states. The states are entangled provided that

$$
p>p_{e}=\frac{1}{(1+4 x)},
$$

while the witness $W^{\prime}$ detects the states provided that

$$
p>p_{w}=\frac{3}{\sqrt{2}(1+4 x)} .
$$

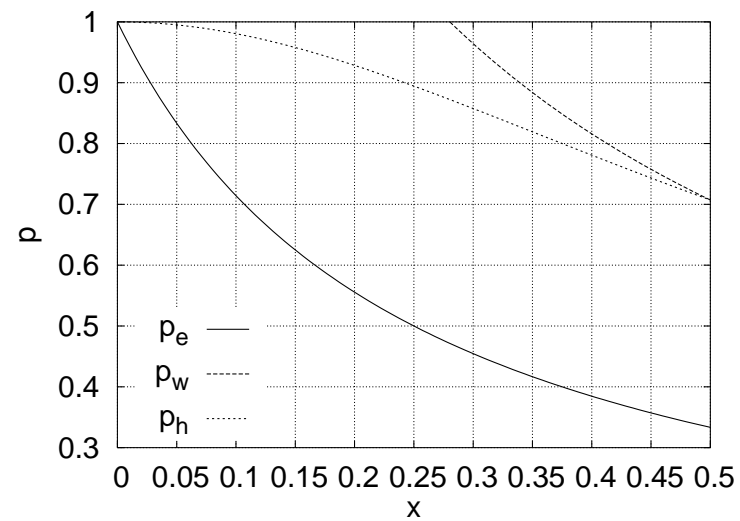

FIG. 2: The graphs show values of $p$ above which the three criteria detect the states $\rho_{p}$ from Eq. (17) depending on $x=$ $a b$. The lowest line corresponds to PPT criterion, the middle line to the Horodecki criterion, and the top line to the shifted witness $W^{\prime}$.

The rhs is larger than or equal to one for $x \leq \gamma$, so that the witness does not detect any states for this range of parameters.

Let us compare the witness with the Horodecki criterion from above [28]: For the states $\rho_{p}$ we have

$$
T_{\rho_{p}}=\left(\begin{array}{ccc}
-2 x p & 0 & 0 \\
0 & -2 x p & 0 \\
0 & 0 & -p
\end{array}\right) .
$$

Since $x \leq 1 / 2$ the states are violating a CHSH inequality if

$$
p^{2}\left(1+4 x^{2}\right)>1 \Leftrightarrow p>p_{h}=\frac{1}{\sqrt{1+4 x^{2}}} .
$$

For $a=b=1 / \sqrt{2}$, when the states correspond to the Werner states [17], both $W^{\prime}$ and the Horodecki criterion detect the states if $p>1 / \sqrt{2}$ which is equivalent to the value found by Werner [17], indicating that $\gamma$ is a sharp bound. For other values of $a$, however, the bounds differ, see Fig. 2. Still, the witness detects a rather large proportion of the states detected by some CHSH inequality.

Let us now consider the general optimal witnesses given by $W=|\phi\rangle\left\langle\left.\phi\right|^{T_{A}}\right.$ with $\left.\mid \phi\right\rangle=\alpha|00\rangle+\beta|11\rangle$. We can rewrite Eq. (9) in the same way as above:

$$
\begin{aligned}
W_{\alpha}= & \frac{1}{4}\left[\mathbb{1} \mathbb{1}+\left(\alpha^{2}-\beta^{2}\right)\left(\sigma_{z} \mathbb{1}+\mathbb{1} \sigma_{z}\right)+(\alpha-\beta)^{2} \sigma_{z} \sigma_{z}\right. \\
& \left.+\frac{\alpha \beta}{\sqrt{2}}\left(\mathcal{B}_{x, y}+\mathcal{B}_{x, z}+\mathcal{B}_{y, z}\right)\right]
\end{aligned}
$$

Again, we would like to find a lower bound for this expression with respect to states not violating a $\mathrm{CHSH}$ inequality. The $\mathrm{CHSH}$ contribution is $\geq-3 \sqrt{2} \alpha \beta$ for states admitting a LHV model.

The expectation value of the other terms $\left(\alpha^{2}-\beta^{2}\right)\left(\sigma_{z} \mathbb{1}+\mathbb{1} \sigma_{z}\right)+(\alpha-\beta)^{2} \sigma_{z} \sigma_{z} \quad$ is $\quad$ bounded 
from below by the minimal eigenvalue. Assuming that $\alpha \geq \beta$, this is given by $-2\left(\alpha^{2}-\beta^{2}\right)+(\alpha-\beta)^{2}$ because $\left(\alpha^{2}-\beta^{2}\right)-(\alpha-\beta)^{2}=2\left(\alpha \beta-\beta^{2}\right) \geq 0$. Hence we obtain for states $\rho_{\text {LHV }}$ obeying all CHSH inequalities the bound

$$
\begin{aligned}
& \operatorname{Tr}\left[W_{\alpha} \rho_{\mathrm{LHV}}\right] \\
& \geq \frac{1}{4}\left(1-2\left(\alpha^{2}-\beta^{2}\right)+(\alpha-\beta)^{2}-3 \sqrt{2} \alpha \beta\right) \equiv-\gamma_{\alpha}
\end{aligned}
$$

which reduces to $\gamma$ from Eq. (15) for $\alpha=1 / \sqrt{2}$. The operator $W_{\alpha}^{\prime}=W_{\alpha}+\gamma_{\alpha} \cdot \mathbb{1}$ is positive on states admitting a local hidden variable model. However, in order to detect some states, it must not be positive on all states. The eigenvector with negative eigenvalue of the witness $W_{\alpha}$ is again the state $\left|\psi^{-}\right\rangle$, and hence also for $W_{\alpha}^{\prime}$, with the eigenvalue $-\alpha \beta+\gamma_{\alpha}$. This is negative for $\alpha \leq[8 /(19-6 \sqrt{2})]^{1 / 2} \approx 0.872$ only, hence $W_{\alpha}^{\prime}$ does not detect any states for a larger value of $\alpha$.

\section{FROM CHSH INEQUALITIES TO OPTIMAL WITNESSES}

Now we address the opposite question: how much can we shift a CHSH witness towards the set of separable states by subtracting the identity operator so that it remains a witness? In other words, for which $\delta>0$ is $2 \mathbb{1}+\mathcal{B}-\delta \mathbb{1}$ still a witness? We calculate $\delta$ depending on the parameters of $\mathcal{B}$ and relate the $\mathrm{CHSH}$ witness to optimal witnesses from a restricted class of witness operators.

First let us parametrize the CHSH operator from Eq. (4) such that all measurements vectors lie in the $x-z$ plane. In particular, we can choose the local coordinate systems such that $\mathbf{a}=\mathbf{b}=\hat{\mathbf{z}}$ and $(\mathbf{a}, \mathbf{b})^{\prime}=$ $\left(\sin \left(\theta_{a, b}\right), 0, \cos \left(\theta_{a, b}\right)\right)$. The operator takes the form

$$
\begin{aligned}
\mathcal{B}= & -s_{a} s_{b} \cdot \sigma_{x} \sigma_{x}+s_{a}\left(1-c_{b}\right) \cdot \sigma_{x} \sigma_{z} \\
& +\left(1-c_{a}\right) s_{b} \cdot \sigma_{z} \sigma_{x}+\left(1+c_{a}+c_{b}-c_{a} c_{b}\right) \cdot \sigma_{z} \sigma_{z},
\end{aligned}
$$

where we abbreviated $\sin \left(\theta_{a, b}\right) \equiv s_{a, b}$ and $\cos \left(\theta_{a, b}\right) \equiv$ $c_{a, b}$. This is already written in the basis of products of Pauli matrices. We can perform a singular value decomposition of the matrix of coefficients and obtain

$$
\begin{aligned}
\mathcal{B} & =\lambda_{+} \tilde{\sigma}_{x} \bar{\sigma}_{x}+\lambda_{-} \tilde{\sigma}_{z} \bar{\sigma}_{z} \\
\lambda_{ \pm} & =\left(2\left(1 \pm \sqrt{1-s_{a}^{2} s_{b}^{2}}\right)\right)^{\frac{1}{2}},
\end{aligned}
$$

where $\tilde{\sigma}_{x, z}$ and $\bar{\sigma}_{x, z}$ are the Pauli operators in rotated bases of party A and B, respectively.

We will now estimate the maximal expectation value that this operator can attain with respect to product states with the help of the following proposition. This will directly provide the desired bound.

Proposition. The maximal expectation value of an operator $\mathcal{A}=\lambda_{x} \sigma_{x} \sigma_{x}+\lambda_{z} \sigma_{z} \sigma_{z}$ with respect to product states is given by $\max \left(\lambda_{x}, \lambda_{z}\right)$. The minimal value is given by $-\max \left(\lambda_{x}, \lambda_{z}\right)$.
Proof. Using the Cauchy-Schwarz inequality, we can estimate

$$
\begin{aligned}
& \left|\left\langle a, b\left|\left(\lambda_{x} \sigma_{x} \sigma_{x}+\lambda_{z} \sigma_{z} \sigma_{z}\right)\right| a, b\right\rangle\right| \\
= & \left|\left\langle\sqrt{\lambda_{x}} \sigma_{x}\right\rangle_{a}\left\langle\sqrt{\lambda_{x}} \sigma_{x}\right\rangle_{b}+\left\langle\sqrt{\lambda_{z}} \sigma_{z}\right\rangle_{a}\left\langle\sqrt{\lambda_{z}} \sigma_{z}\right\rangle_{b}\right| \\
\leq & \sqrt{\left(\lambda_{x}\left\langle\sigma_{x}\right\rangle_{a}^{2}+\lambda_{z}\left\langle\sigma_{z}\right\rangle_{a}^{2}\right)\left(\lambda_{x}\left\langle\sigma_{x}\right\rangle_{b}^{2}+\lambda_{z}\left\langle\sigma_{z}\right\rangle_{b}^{2}\right)} .
\end{aligned}
$$

The maximum of each of the terms in brackets below the square root will surely be attained for vectors in the $x-z$ plane, for which $\left\langle\sigma_{x}\right\rangle^{2}+\left\langle\sigma_{z}\right\rangle^{2}=1$ holds. With the help of Lagrange multipliers, we obtain $\max _{\left[x^{2}+z^{2}=1\right]}\left(\lambda_{x} x^{2}+\right.$ $\left.\lambda_{z} z^{2}\right)=\max \left(\lambda_{x}, \lambda_{z}\right)$. This holds for both terms below the square root. For $\lambda_{x}>\lambda_{z}\left(\lambda_{x}<\lambda_{z}\right)$, the maximum is attained for the eigenstates of $\sigma_{x} \sigma_{x}\left(\sigma_{z} \sigma_{z}\right)$.

Hence the minimal expectation value of the CHSH witness $W_{\mathrm{CHSH}}=2 \mathbb{1}+\mathcal{B}$ with respect to product states is $2-\lambda_{+}$, which follows from the Proposition and from $\lambda_{+} \geq \lambda_{-}$. This means that

$$
\tilde{W}=2 \mathbb{1}+\mathcal{B}-\left(2-\lambda_{+}\right) \mathbb{1}=\lambda_{+} \mathbb{1}+\mathcal{B}
$$

is still a witness. We will show now how this witness can be related to an optimal witness of a restricted class of witnesses that can be written as

$$
W=\sum_{i, j=\{0, x, z\}} c_{i j} \sigma_{i} \otimes \sigma_{j}
$$

which have the property that $W=W^{T}=W^{T_{A}}$. The CHSH witness $W_{\mathrm{CHSH}}=2 \mathbb{1}-\mathcal{B}$ with $\mathcal{B}$ from Eq. (26) belongs to this class, which we will refer to as $\mathrm{EW}_{4}$ in the following. The witnesses of the class $\mathrm{EW}_{4}$ are of special interest from the point of view of quantum key distribution [30]. In these investigations, it was shown that the optimal witnesses of this class are given by

$$
W_{e}=\frac{1}{2}\left(\left|\phi_{e}\right\rangle\left\langle\phi_{e}|+| \phi_{e}\right\rangle\left\langle\left.\phi_{e}\right|^{T_{A}}\right),\right.
$$

where $\left|\phi_{e}\right\rangle$ is an entangled state with real coefficients [30]. Furthermore, it has been shown that the class of witnesses in $\mathrm{EW}_{4}$ can detect states which cannot be detected by the CHSH inequality [31].

Choosing $\left|\phi_{e}\right\rangle$ to be the Bell state $\left|\phi^{+}\right\rangle$in the basis of Eq. (26), the corresponding witness in local form is given by $W_{+}=\left(\mathbb{1}+\tilde{\sigma}_{x} \bar{\sigma}_{x}+\tilde{\sigma}_{z} \bar{\sigma}_{z}\right) / 4$. Then we can write the shifted witness from above as

$$
\tilde{W}=\lambda_{+} \mathbb{1}+\mathcal{B}=4 \lambda_{-} W_{+}+\left(\lambda_{+}-\lambda_{-}\right)\left(\mathbb{1}+\tilde{\sigma}_{x} \bar{\sigma}_{x}\right),
$$

i.e., even after the shift the resulting witness is still given by the sum of an optimal witness from the class $\mathrm{EW}_{4}$ and a positive definite operator. However, if we choose $\theta_{a}=\theta_{b}=\pi / 2$, then $\lambda_{+}=\lambda_{-}=\sqrt{2}$, and the shifted witness $\lambda_{+} \mathbb{1}+\mathcal{B}$ is equal to the optimal witness from the restricted class. Still, the result indicates that the subtraction of the identity operator might not be the optimal strategy for the optimization of the CHSH witness. In the following section, we will use a different approach. 


\section{CHSH INEQUALITIES WRITTEN AS NON-OPTIMAL WITNESSES}

In this section, we show explicitly how any CHSH inequality can be decomposed into a sum of an optimal witness and a general positive operator, starting from the diagonalized CHSH witness. First, we find such decompositions into an optimal witness and a positive operator, and then decompositions involving optimal witnesses $W_{e}$ from the restricted class of witnesses from above.

The Bell operator of Eq. (4) in diagonal form is given by

$$
\begin{aligned}
W_{\mathrm{CHSH}}=2 \cdot \mathbb{1} & +\mu_{+}\left(\left|\psi_{1}\right\rangle\left\langle\psi_{1}|-| \psi_{2}\right\rangle\left\langle\psi_{2}\right|\right) \\
& +\mu_{-}\left(\left|\psi_{3}\right\rangle\left\langle\psi_{3}|-| \psi_{4}\right\rangle\left\langle\psi_{4}\right|\right),
\end{aligned}
$$

where $\mu_{ \pm}=2 \sqrt{1 \pm s_{a} s_{b}}$ and all the eigenstates $\left|\psi_{i}\right\rangle$ for $i=1, \ldots, 4$ are maximally entangled 32 . Choosing convenient local bases, these can be brought to the form

$$
\begin{array}{ll}
\left|\psi_{1}\right\rangle=\left|\phi^{+}\right\rangle, & \left|\psi_{2}\right\rangle=\left|\psi^{-}\right\rangle, \\
\left|\psi_{3}\right\rangle=\left|\tilde{\phi}^{+}\right\rangle, & \left|\psi_{4}\right\rangle=\left|\tilde{\psi}^{-}\right\rangle,
\end{array}
$$

where the local bases of the latter two vectors are different from the local bases of the first two vectors, while all vectors still form an orthonormal set. Note that for $\theta_{a}=\theta_{b}=\pi / 2$, the eigenvalue $\mu_{-}$vanishes, while $\mu_{+}$ reaches its maximal value $2 \sqrt{2}$, so that also $W_{\mathrm{CHSH}}$ has a minimal negative eigenvalue for this choice of settings. In the following, we will refer to these settings as optimal, which is further motivated by the results of the previous section.

We first write the witness $W_{\mathrm{CHSH}}$ directly as the sum of an optimal witness and a positive operator, i.e.,

$$
W_{\mathrm{CHSH}}=\chi|\phi\rangle\left\langle\left.\phi\right|^{T_{A}}+P, \quad P \geq 0, \quad \chi \geq 0,\right.
$$

where $|\phi\rangle$ is an entangled vector. Note that such a decomposition is by no means unique. We start by rewriting

$$
\begin{aligned}
W_{\mathrm{CHSH}}= & 2 \cdot\left(\mathbb{1}-\left|\psi^{-}\right\rangle\left\langle\psi^{-}\right|\right)+\mu_{+}\left|\phi^{+}\right\rangle\left\langle\phi^{+}\right| \\
& +\mu_{-}\left(\left|\tilde{\phi}^{+}\right\rangle\left\langle\tilde{\phi}^{+}|-| \tilde{\psi}^{-}\right\rangle\left\langle\tilde{\psi}^{-}\right|\right) \\
& +\left(2-\mu_{+}\right)\left|\psi^{-}\right\rangle\left\langle\psi^{-}\right|
\end{aligned}
$$

where the two terms in the rhs of the first line are orthogonal to $\left|\psi^{-}\right\rangle$. We use as the entangled vector in Eq. (35) $|\phi\rangle=\alpha|00\rangle+\beta|11\rangle$ with $\alpha \geq \beta$, because the vector corresponding to the negative eigenvalue $-\alpha \beta$ of $|\phi\rangle\left\langle\left.\phi\right|^{T_{A}}\right.$ is $\left|\psi^{-}\right\rangle$. We substitute in the last term of Eq. (36)

$$
\begin{aligned}
\left|\psi^{-}\right\rangle\left\langle\psi^{-}\right|= & -\frac{1}{\alpha \beta}\left[|\phi\rangle\left\langle\left.\phi\right|^{T_{A}}-\alpha^{2} \mid 00\right\rangle\left\langle 00\left|-\beta^{2}\right| 11\right\rangle\langle 11|\right. \\
& \left.-\alpha \beta\left|\psi^{+}\right\rangle\left\langle\psi^{+}\right|\right],
\end{aligned}
$$

arriving at

$$
W_{\mathrm{CHSH}}=\chi|\phi\rangle\left\langle\left.\phi\right|^{T_{A}}+O,\right.
$$

where $\chi:=\left(\mu_{+}-2\right) / \alpha \beta$ and $O$ is given by

$$
\begin{aligned}
O & =2 \cdot\left(\mathbb{1}-\left|\psi^{-}\right\rangle\left\langle\psi^{-}\right|\right)+\mu_{+}\left|\phi^{+}\right\rangle\left\langle\phi^{+}\right| \\
& +\mu_{-}\left(\left|\tilde{\phi}^{+}\right\rangle\left\langle\tilde{\phi}^{+}|-| \tilde{\psi}^{-}\right\rangle\left\langle\tilde{\psi}^{-}\right|\right)+\chi X
\end{aligned}
$$

where $X:=-\alpha^{2}|00\rangle\left\langle 00\left|-\beta^{2}\right| 11\right\rangle\left\langle 11|-\alpha \beta| \psi^{+}\right\rangle\left\langle\psi^{+}\right|$. This is already of the desired form provided that $O$ is a positive operator. An easy bound on the positivity of $O$ can be obtained as follows: we have $X \geq$ $-\alpha^{2}\left(|00\rangle\langle 00|+| 11\rangle\left\langle 11|+| \psi^{+}\right\rangle\left\langle\psi^{+}\right|\right)=$: $Y$. If we denote by $O^{\prime}$ the operator which results from $O$ when we replace $X$ with $Y$, then $O \geq O^{\prime}$, i.e. $O$ will be positive, provided $O^{\prime}$ is. Because $Y=\alpha^{2}\left(\left|\phi^{+}\right\rangle\left\langle\phi^{+}|+| \tilde{\phi}^{+}\right\rangle\left\langle\tilde{\phi}^{+}|+| \tilde{\psi}^{-}\right\rangle\left\langle\tilde{\psi}^{-}\right|\right)$ is proportional to the identity operator in the subspace orthogonal to $\left|\psi^{-}\right\rangle$, we can estimate

$$
\begin{aligned}
O \geq & \left(2-\chi \alpha^{2}+\mu_{-}\right)\left|\tilde{\phi}^{+}\right\rangle\left\langle\tilde{\phi}^{+}\left|+\left(2-\chi \alpha^{2}+\mu_{+}\right)\right| \phi^{+}\right\rangle\left\langle\phi^{+}\right| \\
& +\left(2-\chi \alpha^{2}-\mu_{-}\right)\left|\tilde{\psi}^{-}\right\rangle\left\langle\tilde{\psi}^{-}\right|,
\end{aligned}
$$

so that a sufficient condition for the positivity of $O$ is $\chi \alpha^{2}+\mu_{-} \leq 2$.

Let us investigate the maximal values that $\chi$ and $\alpha$ can attain. First we will maximize $\chi$. Since $\operatorname{Tr}\left[W_{\mathrm{CHSH}}\right]=8=$ $\chi+\operatorname{Tr}[P]$, the decomposition with maximal weight of the partially transposed projector corresponds to a maximal $\chi$. This is bounded by

$$
\chi \leq \frac{2-\mu_{-}}{\alpha^{2}} \leq 2\left(2-\mu_{-}\right) \leq 4,
$$

hence $\chi$ is maximized by choosing $\alpha^{2}=1 / 2$, corresponding to the maximal entangled state. The highest relative weight of $1 / 2$ is reached for the optimal settings, where $\mu_{-}=0$. Maximizing $\alpha$ instead, we obtain the bound

$$
\alpha^{2} \leq \frac{y^{2}}{1+y^{2}} \in\left[\frac{1}{2}, \frac{1}{4-2 \sqrt{2}} \approx 0.854\right]
$$

where $y=\left(2-\mu_{-}\right) /\left(\mu_{+}-2\right)$. The maximal bound is again reached for the optimal settings.

Let us now relate the diagonalized CHSH witness from Eq. (32) to the optimal witnesses of the class $\mathrm{EW}_{4}$, cf. Eq. (30). At this point we can make use of the choice of bases leading to the eigenbasis of the CHSH witness from Eq. (34). Since $\mathbb{1}-\left|\psi^{-}\right\rangle\left\langle\psi^{-}\right|$is the projector onto the symmetric subspace we can rewrite

$$
\begin{gathered}
\mathbb{1}-\left|\psi^{-}\right\rangle\left\langle\psi^{-}\right|=\frac{1}{2}\left(\mathbb{1}+2\left|\phi^{+}\right\rangle\left\langle\left.\phi^{+}\right|^{T_{A}}\right)\right. \\
\Leftrightarrow-\left|\psi^{-}\right\rangle\left\langle\psi^{-}|=| \phi^{+}\right\rangle\left\langle\left.\phi^{+}\right|^{T_{A}}-\frac{\mathbb{1}}{2} .\right.
\end{gathered}
$$

Using this identity, the CHSH witness in the form of Eq. (32) can be written as

$$
W_{\mathrm{CHSH}}=2 \mu_{+} W_{e}+2 \mu_{-} \tilde{W}_{e}+\left(2-\frac{\mu_{+}+\mu_{-}}{2}\right) \mathbb{1},
$$


where $W_{e}=\left(\left|\phi^{+}\right\rangle\left\langle\phi^{+}|+| \phi^{+}\right\rangle\left\langle\left.\phi^{+}\right|^{T_{A}}\right) / 2\right.$, and in analogy for $\tilde{W}_{e}$. This is a good decomposition since, using the abbreviation $x=s_{a} s_{b}$,

$$
2 \geq \sqrt{1+x}+\sqrt{1-x} \Leftrightarrow 4 \geq 2\left(1+\sqrt{1-x^{2}}\right)
$$

is always fulfilled, hence the term proportional to the identity operator is positive or vanishes. From this decomposition we see directly that

$$
W_{\mathrm{CHSH}}-\left(2-\frac{\mu_{+}+\mu_{-}}{2}\right) \cdot \mathbb{1}
$$

is still a witness, but not a CHSH witness anymore. In fact, this bound is equivalent to the bound obtained with the help of the proposition from the last section, because $\mu_{+}+\mu_{-}=2 \lambda_{+}$. Hence the CHSH witness can be written in a very natural way as a superposition of two optimal witnesses from the restricted class $\mathrm{EW}_{4}$ and the identity operator. For the optimal settings, the weight of one of these witnesses vanishes, and we recover the result from the end of the preceding section.

\section{CONCLUSIONS}

In this paper, we investigated the relation between optimal witness operators and the CHSH inequality in detail. We estimated how much optimal witnesses have to be shifted by the identity operator to make them positive on all states admitting a LHV model.

Then we considered the opposite question and obtained tight bounds for which proportion of the identity operator can be subtracted from a CHSH witness, preserving the witness properties. We further related this witness to an optimal witness of the class $\mathrm{EW}_{4}$ of witnesses, which are invariant with respect to partial, as well as complete transposition. The CHSH witness in the parametrization that we used is an element of that class.
Finally, using the diagonalized witness we related it to general optimal witnesses, as well as to optimal witnesses of the class $\mathrm{EW}_{4}$. We found a natural decomposition of the CHSH witness into two such optimal witnesses, and the identity operator. The weight of the identity operator matches the previous results.

A natural next step would be to investigate the relationship between witnesses and more complex Bell inequalities, for instance, the inequality involving three dichotomic measurements per site for two parties found by Śliwa 11. This inequality is of special interest, since is was shown by Collins and Gisin that it can reveal the non-locality of two qubit states which escape the detection via the CHSH inequality [12].

Even more fascinating would be the step to more parties or to systems of higher dimension. Especially the investigation of witnesses for bound entangled states in higher dimensions is of great interest. That could shed light on the Peres conjecture, stating that PPT entangled states do not violate any Bell inequality [33]. If it were possible to show that all Bell inequalities correspond to decomposable witnesses for any choice of the measurements, then the conjecture would be proven. Note that then Bell inequalities with more then two measurements are of special interest, since the CHSH inequality cannot detect PPT states [6]. However, such investigations will become increasingly difficult with the increasing degrees of freedom of the Bell inequalities in higher dimensions.

\section{A. Acknowledgments}

Stimulating discussions with A. Acín, H.J. Briegel, S. Popescu, A. Sanpera, and G. Tóth are gratefully acknowledged. This work was supported by the DFG (SPP 1078, GK 282, and 436POL), and by the EU (QUPRODIS).
[1] J.S. Bell, Physics 1, 195 (1964). Reprinted in J.S. Bell, Speakable and Unspeakable in Quantum Mechanics, (Cambridge University Press, Cambridge 1988).

[2] J.F. Clauser, M.A. Horne, A. Shimony, and R.A. Holt, Phys. Rev. Lett. 23, 880 (1969); Erratum: ibid. 24, 549 (1970).

[3] N.D. Mermin, Phys. Rev. Lett. 65, 1838 (1990).

[4] M. Ardehali, Phys. Rev. A 46, 5375 (1992).

[5] A.V. Belinskii and D.N. Klyshko, Usp. Fiz. Nauk 163(8), 1 (1993).

[6] R.F. Werner and M.M. Wolf, Phys. Rev. A 64, 032112 (2001).

[7] M. Żukowski and Č. Brukner, Phys. Rev. Lett. 88, 210401 (2002).

[8] D. Collins, N. Gisin, N. Linden, S. Massar, and S. Popescu, Phys. Rev. Lett. 88, 040404 (2002).

[9] X.-H. Wu and H.-S. Zong, Phys. Lett. A 307, 262 (2003).
[10] W. Laskowski, T. Paterek, M. Żukowski, and Č. Brukner, Phys. Rev. Lett. 93, 200401 (2004).

[11] C. Śliwa, Phys. Lett. A 317, 165 (2003).

[12] D. Collins and N. Gisin, J. Phys. A 37, 1775 (2004).

[13] S. Popescu and D. Rohrlich, Found. Phys. 24379 (1994).

[14] N. J. Cerf, N. Gisin, S. Massar, and S. Popescu, quant-ph/0410027

[15] N. Brunner, N. Gisin, and V. Scarani, New J. Phys. 7, 88 (2005).

[16] R.F. Werner and M.M. Wolf, Quant. Inf. Comp. 1 (3), 1 (2001).

[17] R.F. Werner, Phys. Rev. A 40, 4277 (1989).

[18] A. Peres, Phys. Rev. Lett. 77, 1413 (1996).

[19] M. Horodecki, P. Horodecki, and R. Horodecki, Phys. Lett. A 223, 1 (1996).

[20] B.M. Terhal, Phys. Lett. A 271, 319 (2000).

[21] M. Lewenstein, B. Kraus, J.I. Cirac, and P. Horodecki, 
Phys. Rev. A 62, 052310 (2000).

[22] M. Barbieri, F. De Martini, G. Di Nepi, P. Mataloni, G.M. D'Ariano and C. Macchiavello, Phys. Rev. Lett. 91, 227901 (2003).

[23] M. Bourennane, M.Eibl, C. Kurtsiefer, S. Gaertner, H. Weinfurter, O. Gühne, P. Hyllus, D. Bruß, M. Lewenstein, and A. Sanpera, Phys. Rev. Lett. 92, 087902 (2004).

[24] J. Barrett, Phys. Rev. A 65, 042302 (2002).

[25] Note, however, that weak Bell inequalities are not required to be valid for all probability distributions (also the correlated ones) which admit a LHV model and are compatible with quantum mechanics. This follows from the above mentioned fact that there are entangled quantum states admitting an LHV model. Thus, weak Bell inequalities are not designed to rule out LHV models.
[26] A. Acín, T. Durt, N. Gisin, and J.I. Latorre, Phys. Rev. A 65, 052325 (2002).

[27] O. Gühne, G. Tóth, P. Hyllus, and H.J. Briegel, quant-ph/0410059

[28] R. Horodecki, P. Horodecki, and M. Horodecki, Phys. Lett. A 200, 340 (1995).

[29] O. Gühne, P. Hyllus, D. Bruß, A. Ekert, M. Lewenstein, C. Macchiavello, and A. Sanpera, Phys. Rev. A 66, 062305 (2002).

[30] M. Curty, M. Lewenstein, and N. Lütkenhaus, Phys. Rev. Lett. 92, 217903 (2004).

[31] M. Curty, O. Gühne, M. Lewenstein, and N. Lütkenhaus, Phys. Rev. A 71, 022306 (2005).

[32] V. Scarani, and N. Gisin, J. Phys. A 34, 6043 (2001).

[33] A. Peres, Found. Phys. 29, 589 (1999). 